\title{
Painting of aluminium panels - state of the art and development issues
}

\author{
Zhenhai Xu, Gerald Anyasodor, and Yi Qin ${ }^{\mathrm{a}}$ \\ Centre for Precision Manufacturing, Dept. of Design, Manufacture \& Engineering Management, \\ The University of Strathclyde, Glasgow G1 1XJ, UK
}

\begin{abstract}
Auto-makers plan a broad shift from steel to aluminium uses over the next decade. In North America, for example, the target is to have $18 \%$ of vehicles having allaluminium bodies by 2025 , in spite of that only less than $1 \%$ has been achieved in 2014 . To achieve this transformation, painting aluminium panels in a mass-production scale with high quality is an important requirement to be met, considering that for long-term protection and surface appearance of the vehicles to be made as well as cost issues. This paper presents a review work carried out on the key elements concerning the painting process, progress in research and applications. Special issues concerning painting aluminium, including the pretreatment of panels surface before e-coat application and the pretreatment of panels before paint baking, are particularly addressed, and remaining issues relating to high quality painting in mass production are discussed.
\end{abstract}

\section{Introduction}

The world is on an unsustainable path regarding oil use and its related environmental impacts such as $\mathrm{CO}_{2}$ emissions. The demands for the automotive energy saving and pollutant emission reduction have been required more than usually expected. Auto-makers are developing better aerodynamics and various new vehicle platforms to minimize the fuel consumption, and light-weighting is another trend due to the fact that a $10 \%$ weight reduction could yield $1.9 \%$ to $8.2 \%$ reduction in fuel consumption [1]. The wide use of lightweight materials in automotive bodies has become an important solution to light-weight structures. Compared with advanced high-strength steels and magnesium alloys, aluminium alloys have a better balance in terms of weight saving, properties and cost, and then they have become an ideal choice [2]. But the difficulty of welding seams and pressing panels into complex shapes has restricted their use to the components that can be cast or formed easily, and a single panel (usually for steel) may need to be redesigned as an assembly of several aluminium alloy sub-parts. At present, aluminium alloys in those forms account only for around $10 \%$ of a typical vehicle's weight, and have almost reached the limit of application due to some technical constraint and economic considerations. For further weight reduction, use of more aluminium-alloy sheets and extrusions for body panels and sub-frames has to be effected. It has been realized largely in a handful of luxury vehicles costing over $\$ 80,000$ and built in a

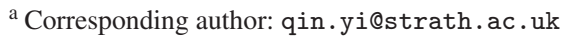

This is an Open Access article distributed under the terms of the Creative Commons Attribution License 4.0, which permits unrestricted use, distribution, and reproduction in any medium, provided the original work is properly cited. 


\section{MATEC Web of Conferences}

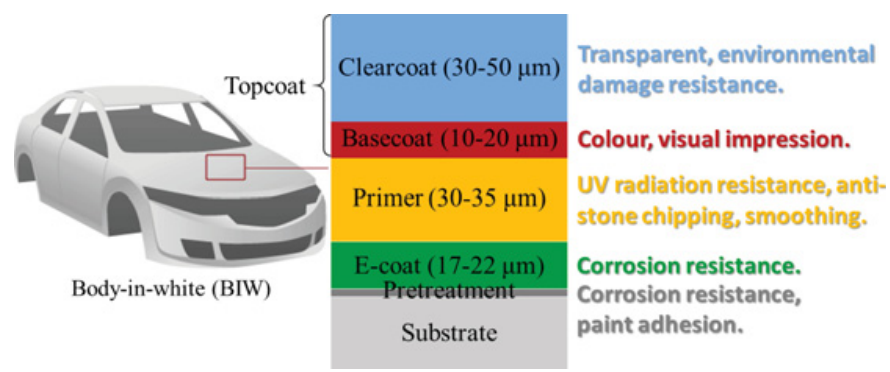

Figure 1. Schematic of a typical four-layer automotive coating system.

modest number. Nevertheless at the end of 2014 Ford Motor Co. rolled out an aluminium-bodied version of its F-150 pickup truck with $15 \%$ weight reduction from its steel predecessor, which will be built in a large volume and has been priced at about $\$ 26,000$ for the base model [3]. A wave of the aluminium alloys use has been kicked off, and auto-makers plan a broad shift from steel to aluminium over the next decade. For example, in North America, it is planned to have all-aluminium bodies reaching $18 \%$ by 2025 from that less than $1 \%$ in 2014 [4].

Use of aluminium alloys is not new to the auto industry. The difference with the F-150, and possibly with future products, is the scale of the increase. It has been driven by the updated or new manufacturing techniques. Ford has spent $\$ 3$ billion to develop the aluminium-bodied trucks and been learning how to make it [3]. Superplastic forming, quick plastic forming and sheet hydroforming can be used to improve the formability of metals, and have been used in the production of some aluminium alloy panels, but the production efficiency needs to be increased. A patented hot-stamping method, named as Solution Heat Treatment, Forming and Cold-die Quenching (HFQ method), is expected to make aluminium alloys easier to work with for auto-makers [5]. To achieve this transformation, painting aluminium panels in a mass-production scale with high quality is an important requirement to be met, considering that for long-term protection and surface appearance of the vehicles to be made as well as cost issues.

This paper presents a review on the key elements concerning the painting process, progress in research and applications as well as a comparison between painting aluminium alloys and painting steel panels. Special issues concerning painting aluminium, including the pretreatment of panels surface before e-coat application and the pretreatment of panels before paint baking, are particularly addressed, and remaining issues relating to high quality painting in mass production are discussed.

\section{General automotive coatings and painting process}

Vehicle production includes four main stages: stamping, bodywork, painting and final assembly. After the first two stages, all parts of a vehicle are attached to the body, which constitutes the core carrier and is called the body-in-white (BIW). Coatings on BIWs applied by the painting require: (1) Outstanding, long-term protection against corrosion, weather, chemical influences, stone chipping; and (2) Perfect surface appearance, including flawlessness, gloss, build, together with evenness and consistency of shade and special effects [6]. Such a multitude of requirements clearly cannot be satisfied by one coating film only, resulting in a multilayer structure of automotive coatings, each of which is responsible for fulfilling the various requirements. A typical automotive coating system consists of four layers as shown in Fig. 1, namely the e-coat, the primer or filler, the basecoat and the clearcoat, and the latter two-layers are combined into the topcoat. Before painting the BIW has to undergo a pretreatment process, which consists of three primary steps: cleaning the vehicle of oils and dirt from the body construction process, deposition of a pretreatment layer, and rinsing of the BIW of pretreatment chemicals. The pretreatment layer, being very thin but having good bonding with substrate, converts the metal surface to a relatively 

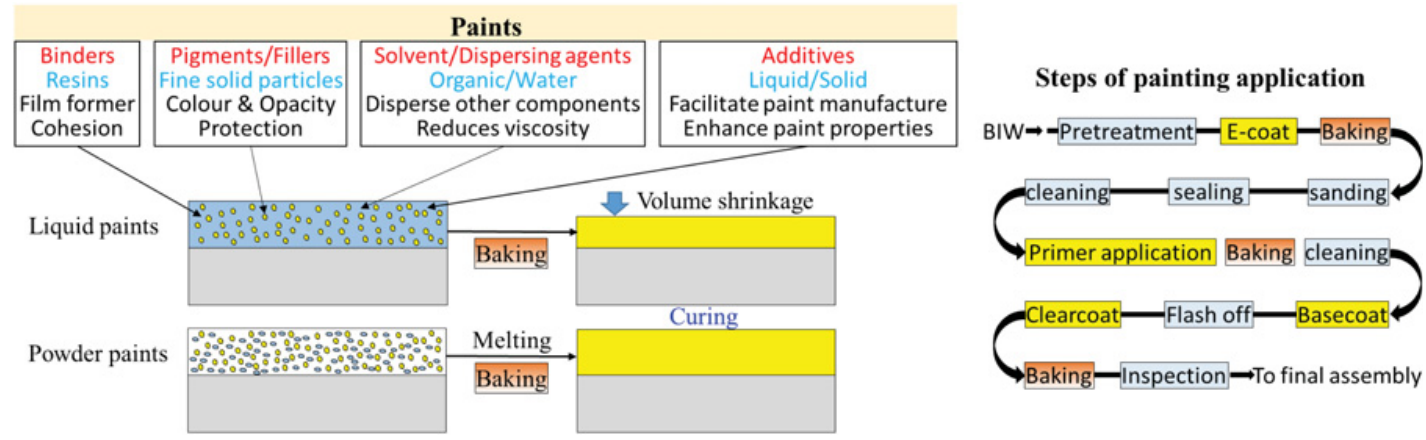

Figure 2. Composition of paints and steps of painting application.

inert material that is less susceptible to chemical attack than an untreated metal, and also provides a consistent micro-porous surface to which the rest of the paint system can adhere [7]. Therefore, the process of painting an aluminium panel is similar to a traditional one for steel panels. Once the e-coat is in place, the primer and topcoat adhere to the panels in, exactly, the same way. Hence, for a paint shop, a major difference in dealing with different materials mainly occurs in the pretreatment stage.

Automotive paints mainly contain four types of raw materials as shown in Fig. 2: binders, pigments or fillers, solvents or dispersing agents, and additives. Owing to their low boiling points and high volatility, solvents can easily seep out of paints during the painting and drying processes, subsequently causing the volatile organic chemicals (VOCs) emission. The painting contributes $90 \%$ of the emission among all stages for vehicle production [8]. About 70-80\% of VOCs escape from the spray booth, while the remaining $15 \pm 5 \%$ is gradually discharged within a dry oven. Two methods are available to reduce VOCs emission: (1) improving paints, such as by decreasing the solvent content, and (2) reducing the VOC emission rate of the flue gas in the stack to allow enough pollutants treating. The former by using waterborne coatings, high-solid coatings, and powder coatings has been on a wider application. However, the powder coating is severely limited as a basecoat for which it is not yet feasible to develop an appropriate range of metallic colours. This obstacle appears to be inherent in the physics of the coating film formation and may never be overcome [9]. The main steps of the painting are also shown in Fig. 2. The e-coat, primer and clearcoat applications are followed by the baking, drying for dispersing agent removal or power melting and cross-linking of paints to form the coating layer. The thermal parameters are $180-190{ }^{\circ} \mathrm{C}$ for $10-20$ minutes, $160-170{ }^{\circ} \mathrm{C}$ for 15-20 minutes and $130-140^{\circ} \mathrm{C}$ for $15-25$ minutes, respectively. While the short intermediate drying of the base coat is called flash off, and typically leads to a solid content of $>90 \%$ in the applied film. The thermal parameters are $23{ }^{\circ} \mathrm{C}$ for $2-5$ minutes for solid rich paints and $50-80^{\circ} \mathrm{C}$ for $3-8$ minutes for waterborne paints [10]. In general, the painting process involves heat treatment for the substrate metals. In the case of the 6xxx series heat-treatable aluminium alloys, used most commonly for automotive outer panels, their ageing peak strength should be reached after these bake ageing. Therefore, the aluminum alloy sheets or stamped panels should be properly pretreated to match the thermal processes of painting for ultimate mechanical strengths.

\section{Special issues concerning painting aluminium}

\subsection{Pretreatment of panels surface before e-coat application}

Trication zinc phosphating has been the standard process worldwide for pretreating multi-metal car bodies for a long time. Zinc phosphate is a crystalline conversion coating that is formed on a metal 


\section{MATEC Web of Conferences}

substrate utilizing the chemical reaction between metal ions that have been dissolved in mineral acids and then diluted with water to form the process solution. It mainly involves three reactions $[10,11]$ :

(1) Picking reaction. When the process solution comes into contact with the metal surface, the mineral acids, mainly nitric acid and phosphoric acid, will dissolve some metal from the surface. This pickling reaction is essential for the coating formation since it can be regarded as a chemical cleaning of the surface, and will affect the adhesion of the coating to the substrate.

(2) Coating reaction. The consumption of hydrogen ions leads to an increase of $\mathrm{pH}$ in the diffusion layer adjacent to the metal surface, exceeding the solubility limits of metal cations, and consequent precipitation of zinc phosphate on the metal surface.

(3) Sludge reaction. The metal ion that is dissolved from the metal surface may be further oxidised by the work solution and will precipitate out as sludge. In the case of steels, $\mathrm{FePO}_{4}$ is deposited. These sludge particles remain in suspension in the bath, and then create rough surfaces, paint adhesion issues and the need for surface sanding and rework later on in the process. Therefore, the sludge has to be continuously removed by filtration techniques in order to maintain constant performance of the conversion layer. Any unfiltered particles could reduce the gloss level achievable after e-coat.

Aluminium panels can be pretreated by the same zinc phosphate with steel ones. It is, however, necessary to modify existing phosphate conditions $[10,12,13]$. Due to its reactiveness, the aluminium surface will react spontaneously with air or water to form aluminium oxide with a thickness of around 1-10 nm, which negatively influences the picking reaction. It requires a stronger etching by adding fluoride to generate amounts of about 50-250 ppm free fluoride, depending on the type of process and application, that is, spraying or dipping. Fluoride also acts as a complex agent to create a phosphate layer of acceptable quality $\left(1.0\right.$ to $\left.1.5 \mathrm{~g} / \mathrm{m}^{2}\right)$. It is also recognised that complex fluorides generally perform better than simple fluorides. The use of fluoride results in sludge formation in the forms of cryolite $\left(\mathrm{Na}_{3} \mathrm{AlF}_{6}\right)$ and elpasolite $\left(\mathrm{K}_{2} \mathrm{NaAlF}_{6}\right)$. As cryolite precipitation in the phosphate film is favoured by both higher sodium and fluoride concentrations, both sodium and free fluoride levels need to be operated below certain limits by applying certain preconditions in terms of flow, composition, and replenishment of the phosphate bath. Systematic studies have shown that a flow of $>0.4 \mathrm{~m} / \mathrm{s}$ parallel to the surface is optimal. And subsequent chemical treatments can allow the use of lower Fluoride levels. Even so, since aluminium generates seven times more sludge per square meter than steel, sludge and surface quality issues quickly develop when the aluminium panels passing through a conventional dip-phosphating process exceeds $20-30 \%$ of the total area treated. The amount of sludge begins to overpower a traditional zinc phosphate system's filtration system, and shortens the effective useful lifetime of the pretreatment baths. A shift in the zinc phosphate from the dipping to spraying can increase the pretreated limit up to $80 \%$. However, the box sections and other interior parts of the car body are difficult to access by spraying, and therefore not properly degreased and covered by the conversion layer. More focuses have been put on the development of the traditional dipping process. It should be noted that BIW are not exclusively constructed of any one metal. Even aluminium intensive vehicles (AIVs) may still contain some steel and other materials. In addition, manufacturers of AIVs may still frequently need to process conventional steel vehicles on the same paint line. Therefore, processing conditions are generally a compromise to provide adequate pre-treatment for all parts of the BIW assembly, not just set up for treating aluminium alone.

Zirconium oxide coatings which has recently been in development by pretreatment suppliers shows a great potential as an alternative to zinc phosphate $[7,12,14]$. It can work well for multi-metal, including aluminium. Figure 3 shows some differences of the two kinds of pretreatments. The zirconium oxide process generates little to no sludge, and is free of phosphorus, resulting in an environment-friendly process. It operates at a significantly lower temperature than zinc phosphate, saving energy used to heat the baths. In addition, it requires fewer process steps, less plant space, reduced maintenance 


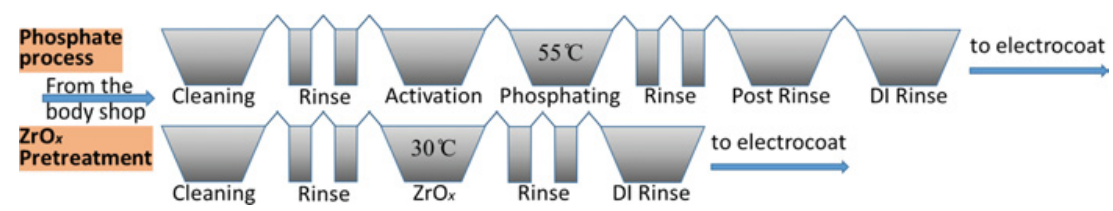

Figure 3. Comparison between different car body pretreatment layouts.

and less consumption of chemicals. Different from the deposited crystalline layer of some 1 to $5 \mu \mathrm{m}$ in thickness of the zinc phosphate, the typical zirconium oxide amorphous coating is approximately $50 \mathrm{~nm}$ in thickness, but performs better. The global supplier of paints and coating PPG Industries has developed a zirconium-based bath system named Zircobond with $80 \%$ sludge reduction [13]. Another adhesive technologies expert Henkel Corporation patented a pretreatment process base on zirconium oxide named BONDERITE Flex to allow the level of aluminium up to $85 \%$ due to the sludge reduction by two-thirds. It reduces the surface etching of aluminium in the traditional step using standard filtration systems and bath agitation rates. The conversion coating on aluminum is then deposited in a second pretreatment stage. This means huge flexibility in the system's ability to handle a wide range of multimaterial combinations without retooling existing paint shops or building new ones [15]. Based on its benefits, zirconium oxide coating pretreatment is increasing rapidly in the marketplace. By 2012, over 60 general industrial dipping and spraying lines converted to zirconium-based pretreatments, and several major North American and European automotive OEM pretreatment lines converted to zirconium pretreatments [16].

\subsection{Pretreatment of panels before paint baking}

The heat-treatable 6xxx alloys sheets used for automotive panels should offer a low and stable strength in the delivered condition for easy stamping and a rapid paint bake hardening response for the high dent resistance. These above thermal processes in painting, mainly three bake cycles, are usually simulated roughly by an ageing treatment at $170-180^{\circ} \mathrm{C}$ for 30 minutes. Painting processes consume $48-60 \%$ of the energy for automobile production [8]. Now an integrated process has been introduced to shorten painting in which the primer and in turn a baking step are eliminated, resulting in an energy saving of around 20\% [17]. Therefore, the low temperature and short time used in the bake ageing treatment is difficult to activate the age hardening potential of alloys completely, and the ability is getting weaker. On the other hand, it is difficult to carry out the artificial paint bake ageing immediately after the solution treatment, therefore it is unable to avoid storage of alloys at room temperature (RT) after the solution treatment, resulting in temper T4 of alloys. Since the precipitation grows due to the natural ageing at RT, the alloy reaches temper T4. It decreases the formability of sheets, and reduces and retards the nucleation and growth of stronger secondary phases, subsequently panels strength is hardly increased by the paint baking but rather decreased. Therefore, a pretreatment before the paint baking should be applied to promote the paint bake response of alloys, and to avoid or compensate for the negative effect of delaying at RT [12]. Pre-ageing has been become a common pretreatment, and is usually applied to alloy sheets in the material making factory as shown in Fig. 4. Re-solution treatment is introduced into alloy sheets before stamping to dissolve the precipitation phase to obtain a high formability. Cold tamping is performed after quenching in a new proposed process [18], while in the newly developed HFQ process hot stamping is conducted at the initial stage of quenching to ensure a higher formability of alloys and to reduce the springback of panels. For these cases, pre-ageing has to be applied to the stamped panels in the forming factory or painting shop before the paint bake, and it challenges the conventional heat treatment processes, favoring a short cycle time and avoiding the distortion of panels. As another pretreatment pre-strain is usually applied to sheets by the uniaxial stretching at RT, 


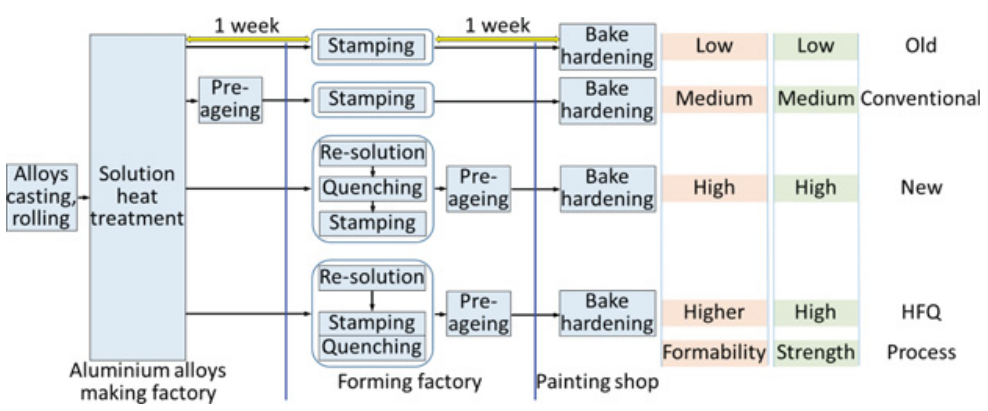

Figure 4. Comparison of old, conventional, new and HFQ processes for heat-treatable aluminium alloy panels.

and it often shows a positive effect on the bake hardening. However, some research indicated that the bake softening resulting from recovery and recrystallization are more prominent [19]. In addition, the typical deformation mode of stamping is a combination of deep drawing and stretching, and then the corresponding strain mode and level of strain in various panels even in different parts of one panel are different from each other. The hot strain in HFQ is also different from the cold strain in other processes shown in Fig. 4. All these factors can affect the pre-ageing process, subsequent bake hardening response, and the mechanical properties uniformity, and therefore need to be further researched systematically.

\section{Conclusions}

The process of painting an aluminium panel may be similar to that for a steel one. A major difference is in the pretreatment stage before the e-coat application. Aluminium panels would need to be further etched due to spontaneous formation of aluminium oxides on the surface. The application of zirconium oxide coatings as an alternative of the zinc phosphate is a solution. To promote paint bake response of aluminium alloys, various types of pre-ageing have to be applied to the sheets or stamped panels, depending on the stamping process. Effect of the mode and level of strain induced from the stamping on the pre-ageing process, subsequent bake-hardening response and mechanical properties uniformity, etc. needs to be further researched systematically. In general, the automotive painting of aluminium panels is progressing, in terms of achieving a balance among cost reduction, environmental compliance and quality improvement.

The study reported in this paper is funded by European Commission through its Seventh Framework Programme (LoCoLite Project, grant agreement No. 604240, for developing "An industrial system enabling the use of a patented, lab-proven materials processing technology for Low Cost forming of Lightweight structures for transportation industries").

\section{References}

[1] R. Wohlecker, M. Johannaber, M. Espig, SAE Technol. 2007-01-0343 (2007)

[2] S.K. Sah, M.A. Bawase, M.R. Saraf, SAE Technol. 2014-28-0025 (2014)

[3] Difference engine: Adding lightness, Web-only article (The Econimist, Los Angeles, 3rd Nov. 2014) http://www.economist.com/news/science-and-technology/21630375-one-wayor-another-fords-new-aluminium-truck-set-be-game-changer-adding-lightness

[4] 2015 North American Light Vehicle Aluminum Content Study, Report (Ducker Worldwide LLC, Jun. 2014) http://www.drivealuminum.org/research-resources/PDF/Research/ 2014/2014-ducker-report 
[5] A. Foster, T.A. Dean, J. Lin, European Patent No. EP2324137, 2013

[6] U. Poth, Automotive Coatings Formulation: Chemistry, Physics and Practices, (Vincentz Network, Hannover, 2008)

[7] S.J. Simko, B. Schneider, J.L. Tardiff, M. Jagner, A. Drews, SAE Int. J. Mater. Manuf. 2, 416 (2009)

[8] J.L. Rivera, T. Reyes-Carrillo, Proc. CIRP, 15, 171 (2014)

[9] C. Chang, C. Lee, Y. Wu, F. Jeng, Resour. Conserv. Recy. 34, 117 (2002)

[10] H. Streitberger, K.D. Ossel, Automotive Paints and Coatings (Wiley-VCH, Weinheim, 2008)

[11] J. Donofrio, Met. Finish., 110, 119 (2012)

[12] The Aluminium Automotive Manual (European Aluminium Association, Brussels, 2015)

[13] R. Schoenberger, Today Motor Veh. Mag. 32 (May 2014)

[14] B. Dunham, D. Chalk, Met. Finish. 110, 112 (2012)

[15] D. Manish, A cleaner, more flexible method to pretreat aluminum, Brochure (Henkel Corporation, 2013) http://www.henkelna.com/us/content_data/308579_Henkel_layout_REV1. pdf

[16] T. Lingenfelter, Environmentally Friendly Pretreatment for Department of Defense Applications, Presentation (ASETSDefense 2012, San Diego, 29 Aug. 2012) http://www .asetsdefense. org/documents/Workshops/ASETS2012/5/2. $\% 20$ Lingenfelter $\% 20 \% 20-\% 20$ For $\% 20$ Web. pdf

[17] BASF Corporation, Eco-Efficiency Analysis: New study conducted by BASF and Dürr regarding automotive OEM coating, Coatings Partner, 3 (Jan. 2013)

[18] K. Mori, S. Maki, M. Ishiguro, Int. Jour. Mach. Tools Manuf. 46, 1966 (2006)

[19] N. Tian, L. Pan, C.C. Liu, J.J. Wang, G. Zhao, L. Zuo, C.M. Liu, Mater. Sci. Forum, 414-419, 706 (2012) 